\title{
Delayed Intrathoracic Gastric Perforation After Obesity Surgery: A Severe Complication
}

\author{
F. Jeroen Vogelaar • Santosh K. Adhin • \\ Herman M. Schuttevaer
}

Received: 22 November 2007 / Accepted: 28 November 2007 / Published online: 15 March 2008

(C) The Author(s) 2008

\begin{abstract}
We describe a case of a patient with an intrathoracic gastric perforation, 6 months after she underwent a gastric banding procedure for the treatment of morbid obesity. After an urgent laparotomy during which the stomach was replaced and oversewn, she recovered uneventfully. The possible mechanism of this severe complication is discussed.
\end{abstract}

Keywords Morbid obesity · Bariatric surgery

Gastric banding · Complication · Gastric perforation

\section{Introduction}

Gastric banding is a frequently performed operation for patients with morbid obesity. Complications have been described extensively. We describe a rare and severe complication of an intrathoracic stomach in a patient with gastric banding, 6 months after the initial procedure, and its treatment.

\footnotetext{
F. J. Vogelaar $(\bowtie) \cdot S$. K. Adhin

Department of Surgery, Rijnland Hospital,

Simon Smitweg 2, P.O. Box 2321 GA,

Leiderdorp, The Netherlands

e-mail: j.vogelaar@rijnland.nl

\section{H. M. Schuttevaer}

Department of Radiology, Rijnland Hospital,

Simon Smitweg 2,

Leiderdorp, The Netherlands
}

H. M. Schuttevaer

Department of Radiology, Leiden University Medical Center,

Leiden, The Netherlands

\section{Case Report}

A 37-year-old woman was admitted to our emergency department with acute onset of abdominal pain, vomiting, and dyspnea. Six months earlier, she had undergone laparoscopic adjustable gastric banding (Lap-Band ${ }^{\circledR}$ )for severe obesity. Physical examination now showed an anxious, dyspneic woman with a respiratory rate of 45 per minute. There was a tachycardia (140/minute) and blood pressure difference between the left $(60 / 40 \mathrm{mmHg})$ and the right arm $(110 / 70 \mathrm{mmHg})$. Auscultation of the chest revealed diminished breathing sounds on the left side. Chest x-ray showed an intrathoracic position of the stomach through a paraesophageal hernia with a shift of the mediastinum to the right (Fig. 1). A small pneumothorax was seen.

After a nasogastric tube was inserted, the patient was intubated because of respiratory insufficiency. A computed tomography (CT) scan of the chest showed a complete atelectasis of the left lung caused by an intrathoracic stomach and a left-sided pneumothorax (Fig. 2). The condition of the patient worsened and she was taken to the operating room for urgent laparotomy.

During laparotomy an ischemic gastric perforation was identified at the orifice of the paraesophageal hernia, with spill of gastric content into the abdomen and thoracic cavity. The intrathoracic stomach was reduced into the abdomen. The gastric perforation was oversewn. The Lap-band was removed, and a Nissen fundoplication was performed. At last, a thoracic drain was placed to treat the pneumothorax.

The patient was treated with intravenous antibiotics (metronidazole and cefuroxime) for 5 days. After 2 days, she was able to leave the Intensive Care Unit. Her 


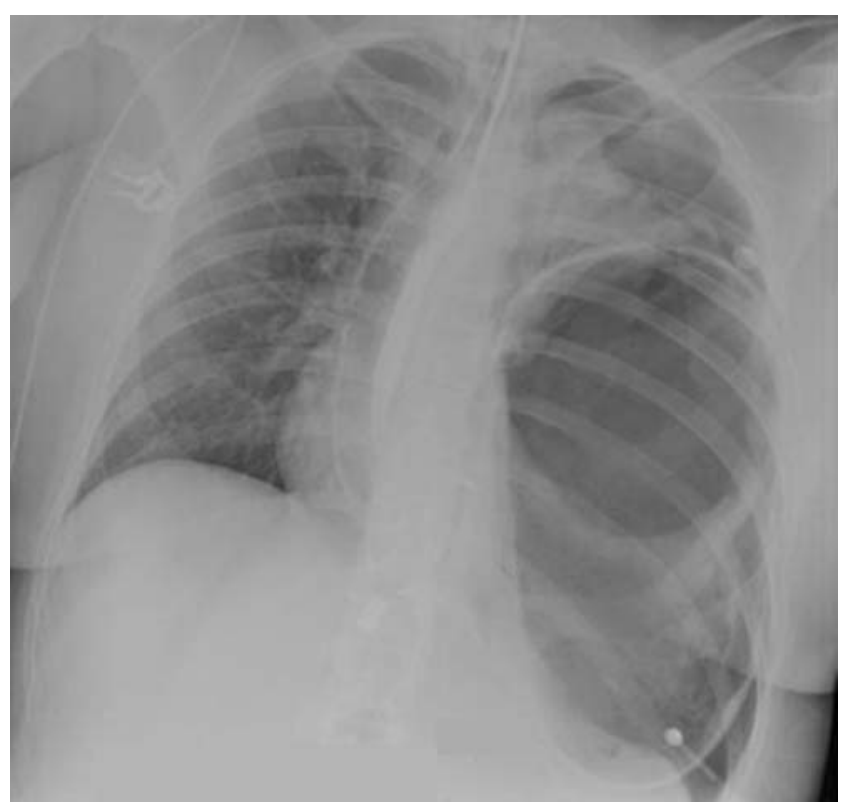

Fig. 1 Intrathoracic position of the stomach with a shift of the mediastinum to the right

postoperative course was uneventful. Thirteen days after surgery, she left the hospital.

\section{Discussion}

Gastric perforation after gastric banding has been described earlier [1]. In all reported cases, the perforation was caused

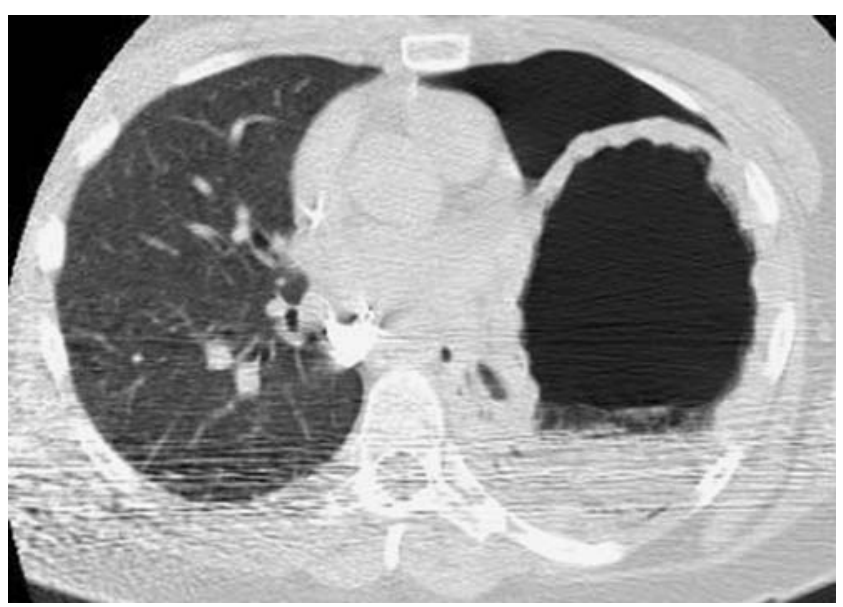

Fig. 2 Complete atelectasis of the left lung caused by an intrathoracic stomach and a left-sided pneumothorax by erosion of the band through the stomach with an incidence of $0.3-3 \%$ of the patients. Furthermore, herniation of the stomach into the thoracic cavity is a well-known entity. It has been described as a late complication after Nissen fundoplication, even in combination with intrathoracic perforation [2-4].

We describe the first case in which both perforation and herniation have occurred simultaneously after obesity surgery and even not as an immediate consequence of the operation. In our case, the gastric band was not the primary cause of the perforation. Most probably, the stomach had moved into the thoracic cavity through a preexistent paraesophageal hernia. Because of air-trapping distal to the band accompanied by having a copious meal, the intrathoracic stomach distended, rotated, and perforated at the orifice of the hernia. The acute onset of pain can be explained by the perforation and the dyspnea appears to be caused by mediastinal shift as well as the pressure atelectasis of the left lung.

Retrospectively, our patient was known to have a paraesophageal hernia before she underwent gastric banding. The possible severe complications of this combination should be considered. A Nissen fundoplication before or in the same operation could be an option for this subgroup of obese patients.

Open Access This article is distributed under the terms of the Creative Commons Attribution Noncommercial License which permits any noncommercial use, distribution, and reproduction in any medium, provided the original author(s) and source are credited.

\section{References}

1. O'Brien PE, Dixon JB. Weight loss and early and late complications - the international experience. Am J Surg. 2002 Dec;184(6B): $42 \mathrm{~S}-5 \mathrm{~S}$.

2. Boggi U, Bellini R, Pietrabissa A, Mosca F. Laparoscopic Nissen fundoplication complicated by late gastroesophageal herniation and intrathoracic perforation: a case report with review of the literature. Surg Laparosc Endosc. 1999 Jan;9(1):57-9.

3. Idani $\mathrm{H}$, Narusue $\mathrm{M}$, Kin $\mathrm{H}$, et al. Acute intrathoracic incarceration of the stomach after laparoscopic Nissen fundoplication. Surg Laparosc Endosc Percutan Tech. 2000 Apr;10(2):99-102.

4. Johansson B, Glise H, Hallerback B. Thoracic herniation and intrathoracic gastric perforation after laparoscopic fundoplication. Surg Endosc. 1995 Aug;9(8):917-8. 